\title{
Pigments Induced by Organomercurial Compounds in Cephalosporium diospyri
}

\author{
By PIA PARN AND R. J. SEVIOUR \\ Department of Botany, Australian National University, \\ Canberra A.C.T. 2600, Australia
}

(Received 3 April I974; revised I July 1974)

\begin{abstract}
SUMMAR Y
The major pigments induced by parahydroxymercuribenzoate and parachloromercuribenzoate (both $\mathrm{SH}$ group inhibitors) in the fungus Cephalosporium diospyri have been characterized as carotenoids. Those present in both chemicallyinduced and light-induced cells include phytoene, $\beta$-carotene, $\gamma$-carotene, lycopene, torulene and neurosporaxanthin. Two other organomercurial compounds, phenyl mercuric acetate and phenyl mercuric chloride, were also found to induce pigment formation. These pigments have the absorption properties of carotenoids, but have not been fully characterized. A range of other SH group inhibitors tested failed to induce any pigment formation.
\end{abstract}

\section{INTRODUCTION}

Many heterotrophic micro-organisms respond to light by increased synthesis of carotenoid pigments (Batra, 1972). Rau (1967) first reported that parahydroxymercuribenzoate (PHMB) or parachloromercuribenzoate (PCMB) could substitute for light in carotenogenesis in Fusarium aquaeductum, and Batra (1967) showed that antimycin A was able to mimic light in carotenoid production in Mycobacterium marinum. More recently, hydrogen peroxide was reported to substitute for light in $F$. aquaeductum (Theimer \& Rau, I970). These compounds do not induce the same response in all light-sensitive fungi. Neither antimycin A nor hydrogen peroxide was effective in either Verticillium agaricinum (Valadon \& Mummery, I97I $a$; Mummery \& Valadon, 1973) or Cephalosporium diospyri (Seviour \& Codner, I973), although both fungi synthesize carotenoids in response to light. Furthermore, far from stimulating carotenoid production in the dark in $M$. marinum, PHMB inhibited normal synthesis in the light (Batra, Gleason \& Jenkins, 1969).

The role of PCMB and PHMB in carotenoid synthesis in C. diospyri and $V$. agaricinum is not resolved. Seviour (1970) noticed that in the presence of these compounds, agar-grown colonies of $C$. diospyri synthesized a salmon-pink water-insoluble pigment in the dark. The colonies closely resembled those containing carotenoids synthesized under illumination, but the PCMB- and PHMB-induced pigments were not characterized. The assumption that this pigmentation was carotenoid in nature was criticized by Valadon \& Mummery (I97I $a$ ), because of observations that, under similar cultural conditions, $V$. agaricinum synthesized a pigment they suggested was a naphthaquinone. The present study was undertaken in an attempt to resolve this question by identifying the PCMB/PHMB-induced pigments in $C$. diospyri and comparing the pigment composition with that of light-treated cells, extending an earlier study on the identification of these carotenoids (Codner \& Platt, 1959). 


\section{METHODS}

Organism. Cephalosporium diospyri (ATCC9066), described by Crandall (1945), was reclaimed from a soil culture originally obtained from the University of Bath culture collection.

Growth conditions for pigment production. For the production of light-induced carotenoids, C. diospyri was grown in submerged liquid culture in $50 \mathrm{ml}$ amounts of $2 \%(\mathrm{v} / \mathrm{v})$ malt extract broth (Oxoid CM 57) in $250 \mathrm{ml}$ Erlenmeyer flasks in a Gallenkamp orbital incubator (280 rev./min) at $25{ }^{\circ} \mathrm{C}$, illuminated continuously from above with four 20 Watt fluorescent lights yielding approx. $5 \times 10^{3}$ lux at the surface of the cultures. After Io days, mycelium was harvested by centrifugation, washed and freeze-dried.

Submerged liquid culture techniques could not be used for the production of PHMB/ PCMB-induced pigments, since these compounds are ineffective under such conditions (Seviour \& Codner, 1973). After preparation of malt extract agar (Oxoid CM 59) plates containing between $1 \mathrm{IO}^{-5}$ and $5 \times 1 \mathrm{IO}^{-4} \mathrm{M}-\mathrm{PCMB}$ or PHMB, a sterile membrane filter (Gelman Metricel, $0.45 \mu \mathrm{m}, 47 \mathrm{~mm}$ diam) was placed on the agar, and the fungus inoculated on to this membrane, allowing the whole colony to be detached cleanly. After preliminary tests to ascertain the optimal concentrations of PCMB/PHMB to use for carotenoid production, about 300 plates were prepared for each compound, inoculated, and incubated in the dark at $25{ }^{\circ} \mathrm{C}$ for $2 \mathrm{I}$ days. The resulting colonies were bulked and freeze-dried for pigment analysis.

PHMB as its sodium salt and as a $0.05 \mathrm{M}$ solution in glycylglycine buffer was supplied by Sigma, and PCMB as its sodium salt by Calbiochem.

Extraction of the pigments. Carotenoids were repeatedly extracted with acetone from lightgrown cultures of $C$. diospyri until the extracts were colourless. The combined extracts were saponified and partitioned into epiphasic and hypophasic fractions essentially as described by Davies (1965). Sterols were removed by centrifugation after storing extracts at $-20^{\circ} \mathrm{C}$. The chemically-induced pigments were extracted and analysed in the same manner at $-20^{\circ} \mathrm{C}$. The chemically-induced pigments were extracted and analysed in the same manner as those from light-grown cultures.

Purification of epiphasic pigments. The total epiphasic fraction from light-, PCMB- and PHMB-induced mycelium was chromatographed on Whatman No. I paper impregnated with aluminium hydroxide equivalent to $7.5 \%(\mathrm{w} / \mathrm{w})$ aluminium oxide (Whatman Chromedia $\mathrm{AH}$ 8I paper) as used by Valadon \& Mummery (I97 $\mathrm{I} b$ ), but run in ascending direction with $n$-hexane as developing solvent. The less polar components were resolved into one colourless zone and usually seven coloured zones (fractions 2 to 8 ), but separation of the small amounts of the several polar components was poor. All bands were eluted with acetone, with the exception of the slowest-moving pink zone in each extract. This was recovered with $5 \%(\mathrm{v} / \mathrm{v})$ acetic acid in diethyl ether (Goodwin, 1952) and finally partitioned into $n$-hexane, where it was stored together with the other fractions under nitrogen at $-\mathrm{I} 5{ }^{\circ} \mathrm{C}$. The absorption spectra given by each of these fractions showed that many were still mixtures, and so each was re-chromatographed on thin layers of silica gel G (Merck) developed with appropriate solvents. Fractions 2 to 8 were run with increasing concentrations of acetone ( $\mathrm{I}$ to $5 \%, \mathrm{v} / \mathrm{v}$ ) and benzene ( 5 to $20 \%, \mathrm{v} / \mathrm{v}$ ) in light petroleum, and the polar components with increasing concentrations of acetone ( 5 to $10 \%, v / v)$ in $n$-hexane. Separated components from these were again eluted with acetone, and finally transferred to $n$-hexane. In most cases each original fraction was resolved into more than one component. However, some of these were subsequently identified as being in more than one of the original 
zones, as a result of the initial incomplete separation. Therefore, to clarify the presentation of results each final component will be referred to by suffix (e.g. original zone resolved into two components referred to as $2 \mathrm{a}$ and $2 \mathrm{~b}$, etc.).

Chromatographic separation of hypophasic pigments. Pigments were partitioned into benzene using a modification of the technique described by Rau \& Zehender (1959), and finally dissolved in $n$-hexane. Individual components were separated by t.l.c. on cellulose adsorbent (Merck), developed with light petroleum (b.p. 40 to $60^{\circ} \mathrm{C}$ ), and eluted with acetone.

Determination of spectra. Absorption spectra were determined in various solvents with a Varian Techtron $635 \mathrm{M}$ recording spectrophotometer. Mass spectra were obtained by a direct-insertion probe method in a GEC-AEI MS902 instrument at $70 \mathrm{eV}$ and a temperature gradient of 100 to $280^{\circ} \mathrm{C}$. As a high-resolution technique was involved, and only small quantities of pigments were available, results were recorded on a computer print-out showing both mass peak data and empirical formulae.

Quantification of pigments. Pigments were quantified (Davies, 1965) and each expressed as a percentage of the total. Values of $E_{1 \mathrm{~cm}}^{\mathrm{o}}$ for each were obtained from the literature (Goodwin, 1955; Davies, 1965), and an $E_{1 \mathrm{~cm}}^{1 \%}$ of 2500 used to calculate total pigment content.

\section{RESULTS}

\section{Pigment production in response to $P C M B$ and $P H M B$}

PCMB and PHMB (in either aqueous or buffer solution) were only effective as pigment inducers over a very narrow concentration range. In both cases, maximal pigment production occurred at a final concentration of $5 \times 10^{-4} \mathrm{M}$, but colony growth was severely inhibited. No pigment induction was noticed with either compound at concentrations lower than $5 \times 10^{-5} \mathrm{M}$, which is higher than the minimal level required for $F$. aquaeductum (Rau, 1967). To produce pigments for analysis a $2.5 \times 10^{-4} \mathrm{M}$ concentration was chosen.

Pigmentation of colonies was non-uniform, only those parts of the fungus in contact with the agar surfaces being coloured: consequently much less pigment was obtained per unit of tissue with these chemicals than with light, mainly because the non-pigmented areas of tissue were included in the sample analysed. Although PCMB and PHMB are in the same ionic form in solution (Sigma Technical Bulletin No. 300), the action of these two compounds on $C$. diospyri differed: PHMB produced a much greater inhibitory effect upon growth than did PCMB, and was less effective (approx. $50 \%$ ) in inducing pigment synthesis.

\section{A general comparison between light- and chemically-induced total pigments}

Pigments of both light- and chemically-induced cultures showed certain common features. They behaved identically during the saponification and solvent-partitioning procedures, and absorption spectra of crude total pigment extracts from light- or chemicallyinduced cells all showed the three- or four-peak spectra characteristic of carotenoids (Table I). Hypochromic shifts in benzene and carbon disulphide were apparent for all three extracts, and such shifts are also characteristic of carotenoids (Davies, 1965). These preliminary results suggested that the chemically-induced pigments were carotenoid in nature, and the major individual components were characterized further. 


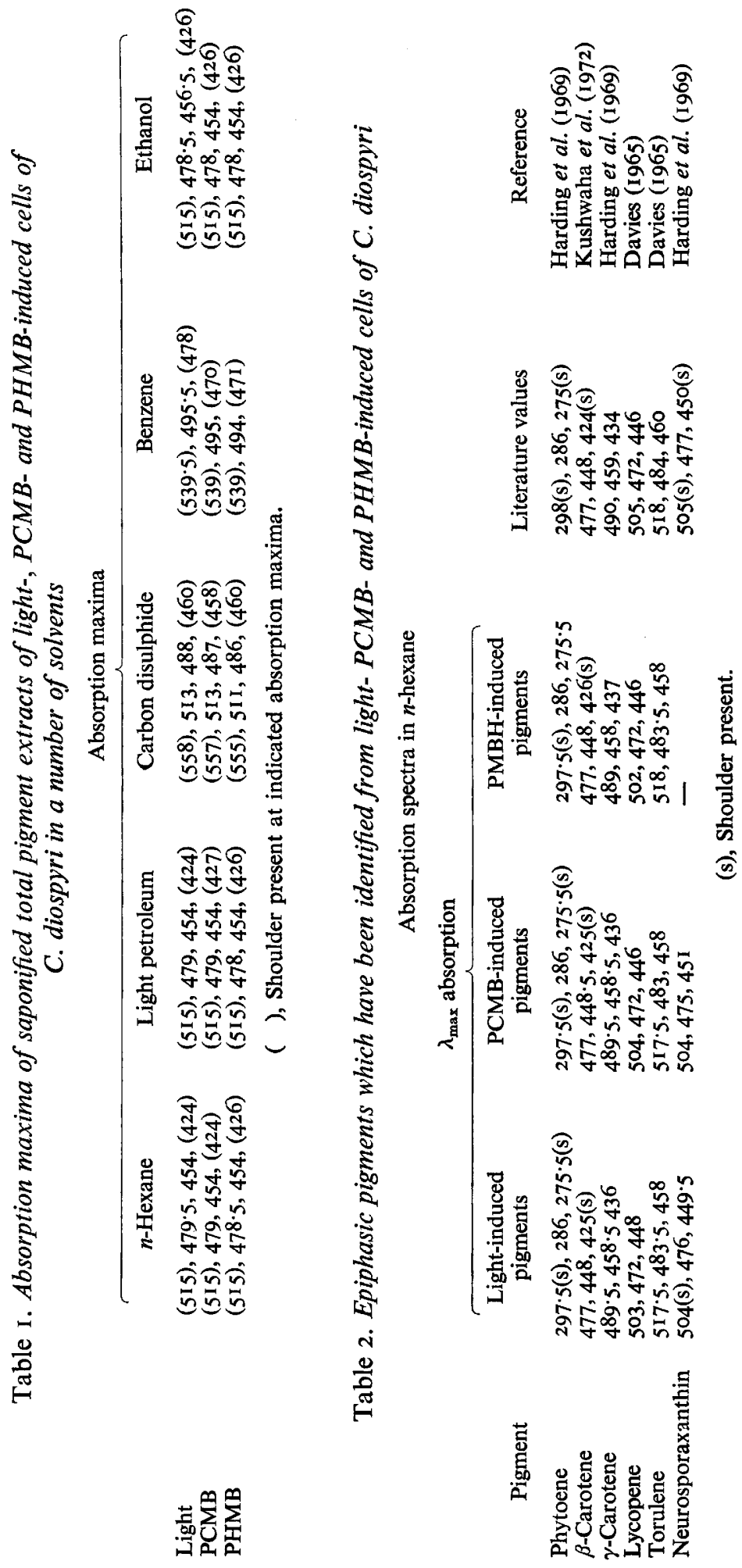




\section{Pigment composition of $C$. diospyri}

Carotenoids identified from light-grown cells will be dealt with consecutively, and comparisons with chemically-induced pigments made where possible. The $\lambda_{\max }$ for all fractions identified are summarized in Table 2.

Phytoene. The colourless fractions I obtained from paper chromatography of all three treatments were not purified further. All had very similar absorption spectra, and $\lambda_{\max }$ which corresponded to published values for phytoene (Harding, Huang \& Mitchell, 1969). As with most separated components, mass spectral analysis was not possible with chemicallyinduced fractions owing to insufficient recovery of pigment. However, a spectrum for light fraction I showed a molecular ion peak at $\mathrm{m} / \mathrm{e} 544$ (mol. wt 544), necessary for authentic phytoene (empirical formula $\mathrm{C}_{40} \mathrm{H}_{64}$ ). The fragmentation pattern was also very similar to published spectra of phytoene (e.g. Kushwaha, Pugh, Kramer \& Kates, 1972). However, no fluorescent bands or absorption spectra characteristic of phytofluene were detectable in any of the colourless fractions.

$\beta$-Carotene. Fractions 2 a from all three treatments gave $\lambda_{\max }$ showing close agreement with published values for $\beta$-carotene (Kushwaha et al. 1972). Mass spectral analysis of light-induced pigment 2 a showed a parent ion at $\mathrm{m} / \mathrm{e} 536\left(\mathrm{C}_{40} \mathrm{H}_{56}\right.$, mol. wt 536). Characteristic ion peaks at $\mathrm{m} / \mathrm{e} 444(\mathrm{M}-92)$ and $\mathrm{m} / \mathrm{e} 430(\mathrm{M}$-106) are consistent with $\beta$-carotene (Kushwaha et al. 1972). The mass spectrum of the PCMB-induced fraction $2 \mathrm{a}$ also contained the molecular ion $\left(\mathrm{M}^{+}\right)$peak at 536 , although in this case, due to the limited quantities of pigment, only an incomplete fragmentation pattern was obtained.

$\gamma$-Carotene. Absorption spectra of light $4 \mathrm{a}$, PCMB $3 \mathrm{~b}$ and PHMB $4 \mathrm{a}$ fractions had $\lambda_{\max }$ suggesting $\gamma$-carotene. When these three fractions were co-chromatographed on silica gel $\mathrm{G}$ in light petroleum (b.p. 40 to $60^{\circ} \mathrm{C}$ ) containing increasing concentrations of acetone, they failed to separate, providing further evidence of their similarity.

Lycopene. From $\lambda_{\max }$ values of absorption spectra of light 5 b, PCMB 5 a and PHMB 5 a, these fractions were identified as lycopene (Davies, 1965). Again, co-chromatography using increasing concentrations of acetone in light petroleum failed to separate them. No mass spectral data could be obtained.

Torulene. Values of $\lambda_{\max }$ for the three 7 and 8 fractions agreed closely with published values for torulene (Davies, 1965). In addition, a mass spectrum of the light-induced sample showed a parent $\mathrm{M}^{+}$peak at 534, representing an empirical formula of $\mathrm{C}_{40} \mathrm{H}_{54}$ which corresponds to torulene. No mass spectral data were obtainable from the chemically-induced pigments. However, co-chromatography of all samples using increasing concentrations of benzene in light petroleum failed to produce any further separation.

Neurosporaxanthin. The hypophasic pigments were resolved into two major components on silica gel G. In both light- and PCMB-induced cell extracts, the faster (pink) band had $\lambda_{\max }$ at 504,476 and $450 \mathrm{~nm}$ in $n$-hexane. These values agree well with published data for neurosporaxanthin (Harding et al. 1969). No well-defined spectrum was obtained with the corresponding PHMB fraction, so it was not identified. The slower-running yellow fraction present in all samples gave a poor absorption spectrum and remains unknown.

Results (Table 3 ) show that the relative proportions of identified carotenoids were very similar between both light- and chemically-induced cells.

Other epiphasic pigments. In addition to the major pigments discussed above, $C$. diospyri contained a number of other carotenoids. In most cases, they were present in trace amounts and were not obtained spectroscopically pure. These have not been identified. However, in a few instances spectra were obtained, and from these it was possible tentatively to identify 
Table 3. Amounts of the major carotenoids present in light-, PCMB- and PHMB-induced cells of C. diospyri

\begin{tabular}{|c|c|c|c|c|c|}
\hline \multirow[b]{2}{*}{ Pigment } & \multirow[b]{2}{*}{$E_{1 \mathrm{~cm}}^{1 \%}$} & \multirow[b]{2}{*}{ Reference } & \multicolumn{3}{|c|}{ Percentage of total extract } \\
\hline & & & Light & PCMB & PHMB \\
\hline Phytoene & 1250 & Davies (1965) & 14.90 & $10 \cdot 60$ & $14 \cdot 90$ \\
\hline$\beta$-Carotene & 2580 & Goodwin (1955) & $27 \cdot 30$ & 23.00 & $23 \cdot 40$ \\
\hline$\gamma$-Carotene & 3100 & Davies (1965) & $4 \cdot 45$ & $2 \cdot 47$ & $2 \cdot 32$ \\
\hline Lycopene & 3450 & Davies (1965) & 0.75 & 0.98 & $1 \cdot 90$ \\
\hline Torulene & 2780 & Davies (1965) & $2 I \cdot 30$ & $26 \cdot 10$ & II 70 \\
\hline Neurosporaxanthin & 1715 & Harding et al. (I969) & $16 \cdot 80$ & $15 \cdot 90$ & - \\
\hline
\end{tabular}

the following carotenoids in both light- and chemically-induced cells: $\zeta$-carotene $\left(\lambda_{\max }\right.$ in $n$-hexane at 376,399 and $425 \mathrm{~nm}$ ), running just behind $\beta$-carotene on thin layers of silica gel G; neurosporene $\left(\lambda_{\max }\right.$ at $4 \mathrm{I} 6,439$ and $\left.466 \mathrm{~nm}\right)$, running between $\gamma$-carotene and lycopene; and a pigment with $\lambda_{\max }$ at 450,423 and $400 \mathrm{~nm}$ which is similar to published values for $\alpha$-zeacarotene, but with different chromatographic behaviour, running behind lycopene on silica gel G (Davies, 1965).

\section{Further evidence for the carotenoid nature of the chemically-induced pigments}

Diphenylamine (DPA), which inhibits light-induced carotenoid production in C. diospyri (Seviour \& Codner, I973), also inhibited the chemically-induced pigment synthesis. This was illustrated using 'lawn sown' plates of $C$. diospyri in which two wells had been cut, one containing $1 \mathrm{IO}^{-2} \mathrm{M}$-DPA, the other $5 \times 10^{-3} \mathrm{M}-\mathrm{PCMB}$ or -PHMB. After dark incubation, pigmentation occurred as a narrow ring around the wells containing PCMB or PHMB, except on the side adjacent to the well containing DPA, where pigmentation was inhibited.

\section{Other chemical inducers of pigmentation}

Because the inhibitory properties of PCMB and PHMB might implicate SH groups in the induction process ( $\mathrm{Rau}, \mathrm{I967}$ ), a wide range of other $\mathrm{SH}$ group inhibitors, including both organic and inorganic mercury-containing compounds, were tested. In addition, parahydroxybenzoate (PHB) and 3,4-dihydroxybenzoate (DHB), both possible intermediates in microbial degradation of PCMB or PHMB (Furukawa \& Tonomura, 197I), were tried. Of all the compounds tested, only phenylmercuric chloride (PMC) and phenylmercuric acetate (PMA) induced detectable amounts of pink pigment in $C$. diospyri, and then only when grown on solid medium. The most effective concentration for both compounds was $2.5 \times 10^{-4} \mathrm{M}$, on malt extract agar. Neither compound has previously been reported as having photomimetic properties. However, the pigments appeared to be carotenoid, and an absorption spectrum of extracts from PMC-induced cells showed a typical carotenoid three-peak spectrum between 400 and $500 \mathrm{~nm}$. Absorption maxima occurred at different wavelengths in crude extracts of light- and PCMB/PHMB-induced cells ( $\lambda_{\max }$ in $n$-hexane at 428,460 and $483 \mathrm{~nm}$ ), suggesting significant differences in their carotenoid composition. Because only small quantities of pigment were available, further characterization was not attempted.

\section{DISCUSSION}

A combination of analytical techniques has provided convincing evidence that the pigments induced in Cephalosporium diospyri by PCMB and PHMB are carotenoids, identical to those produced in response to light in this organism. None of the data obtained supported claims 
that these pigments might be naphthaquinones (Valadon \& Mummery, 1971a). Although only the major pigment components were identified, $C$. diospyri has a very similar carotenoid composition to other light-inducible fungi such as Neurospora crassa (Harding et al. 1969), Verticillium agaricinum (Valadon \& Mummery, 197.1 $a$ ) and Fusarium aquaeductuum (Bindl, Lang \& Rau, 1970). Quantitative studies (Table 3) have shown that in both light- and chemically-induced cells of $C$. diospyri, the carotenoids present in largest amounts are those which are considered to be end products in the biosynthetic pathway (Goodwin, I97I). Pigments thought to be intermediates were present in much smaller amounts. This suggests that the various carotenoids, regardless of the nature of the inducer, are synthesized via the same biosynthetic pathway which occurs in other tissues.

The observation that PMA and PMC also induce pigment synthesis in $C$. diospyri is interesting. These pigments have the spectral characteristics of carotenoids and, by this criterion, are seen to be quite different from the pigment $\mathrm{I}, 4,5,8$ tetrahydroxyanthraquinone produced in response to PMA in a mercury-resistant strain of Pyrenophora avenae (Greenaway, 197I). With this finding, some pattern begins to emerge concerning the structural features required of compounds for them to induce pigment production in C. diospyri. All four effective chemicals are aromatic organomercurial compounds. By contrast, several other aliphatic mercury compounds were ineffective, as were a number of inorganic mercury compounds. Furthermore, as neither PHB nor DHB induced pigmentation, the possibility that a phenyl or benzoate group may be solely responsible, either directly or after metabolic conversion, can probably be discounted. Although a search for further inducers among organomercurial compounds of this type may prove rewarding, the failure of other structurally similar organomercurial compounds tested for photomimetic effect (e.g. parahydroxymercuriphenyl sulphonate) implies that other factors may be significant. Of these, membrane permeability is likely to be important, since it is known that organic and inorganic mercurial compounds differ in their ability to cross cell membranes (Scott, Knight, Settlemire \& Brierly, 1970; Selwyn, 1972).

Further understanding of the mechanism of induction by these chemicals will probably have to await the development of a more suitable experimental system than agar-grown cultures of $C$. diospyri. However, this organism, which produces no coloured carotenoids in the dark, is likely to prove a more informative organism for resolving light-regulated carotenoid synthesis than are fungi which produce small quantities of carotenoids even before exposure to light.

We thank Dr G. A. Chilvers for his encouragement during this study, and both he and Dr A. E. Ashford for their constructive criticism of the manuscript. We also thank $\mathrm{Mr} \mathrm{C}$. Macdonald for assistance with the mass spectroscopy.

\section{REFERENCES}

BATRA, P. P. (1967). Mechanism of photoinduced carotenoid synthesis. Induction of carotenoid synthesis by antimycin $\mathrm{A}$ in the absence of light in Mycobacterium marinum. Journal of Biological Chemistry 42 , $5630-5635$.

BAtra, P. P. (1972). Mechanism of light induced carotenoid synthesis in non-photosynthetic plants. In Photophysiology, vol. 6, pp. 47-76. Edited by A. C. Giese. New York: Academic Press.

Batra, P. P., Gleason, R. M. \& Jenkins, J. (1969). Mechanism of photo-induced and antimycin A-induced carotenoid synthesis in Mycobacterium marinum. Requirements for carotenogenesis and further evidence for protein synthesis following induction. Biochemica et biophysica acta 177, 124-1 35 . 
BINDL, E., LANG, W. \& RAU, W. (1970). Untersuchungen über die lichtabhängige Carotinoidsynthese. VI. Zeitlicher Verlauf der Synthese der einzelnen Carotinoide bei Fusarium aquaeductuum unter verschiedenen Induktionsbedingungen. Planta 94, I 56-I 74 .

Codner, R. C. \& Platt, B. C. (1959). Light induced production of carotenoid pigments by Cephalosporia. Nature, London 184, 74I-742.

Crandall, B S. (1945). A new species of Cephalosporium causing Persimmon wilt. Mycologia 37, 495-498.

DAvies, B. H. (1965). Analysis of carotenoid pigments. In Chemistry and Biochemistry of Plant Pigments, pp. 489-532. Edited by T. W. Goodwin. London: Academic Press.

FURUKAWA, K. \& TONOMURA, K. (1971). Enzyme systems involved in the decomposition of phenyl mercuric acetate by mercury-resistant Pseudomonas. Agricultural Biological Chemistry 35, 604-6Io.

Goobwin, T. W. (1952). Studies in Carotenogenesis. III. Identification of the minor polyene components of the fungus Phycomyces blakesleeanus and a study of their synthesis under various cultural conditions. Biochemical Journal 50, 550-558.

Goodwin, T. W. (I955). Carotenoids. In Modern Methods of Plant Analysis, vol. 3, pp. 29I-305. Edited by K. Paech and M. V. Tracey. Berlin: Springer-Verlag.

GooDwin, T. W. (I97I). Biosynthesis. In Carotenoids, pp. 577-636. Edited by O. Isler. Basel: Birkhäuser Verlag.

GreenawAY, W. (1971). Relationship between mercury resistance and pigment production in Pyrenophora avenae. Transactions of the British Mycological Society 56, 37-44.

Harding, R. W., Huang, P. C. \& Mitchell, H. K. (1969). Photochemical studies of the carotenoid biosynthetic pathway in Neurospora crassa. Archives of Biochemistry and Biophysics 129, 696-707.

Kushwaha, S. C., Pugh, E. L., Kramer, J. K. G. \& Kates, M. (I972). Isolation and identification of dehydrosqualene and $\mathrm{C}_{40}$-carotenoid pigments in Halobacterium cutirubrum. Biochimica et biophysica acta 260, 492-506.

Mummery, R. S. \& Valadon, L. R. G. (1973). The effect of antimycin A on carotenogenesis in Verticillium agaricinum. Planta 109, 353-356.

RAU, W. (1967). Untersuchungen über die lichtabhängige Carotenoidsynthese. II. Ersatz der Lichtinduktion durch Mercuribenzoat. Planta 75, 263-277.

RAU, W. \& ZeHENDER, C. (1959). Die Carotinoide von Fusarium aquaeductuum Lagh. Archiv für Mikrobiologie 32, 423-428.

ScotT, K. M., KNIGHT, V. A., SetTlemire, C. T. \& Brierley, G. P. (I970). Differential effects of mercurial reagents on membrane thiols and on the permeability of the heart mitochondrion. Biochemistry 9 , 714-721.

SelWyn, J. (1972). Permeability effects of organomercurials. Biochemical Journal 130, $65 \mathrm{P}-67 \mathrm{P}$.

Seviour, R. J. (1970). Effect of light on Cephalosporium diospyri. Ph.D. thesis, Bath University of Technology.

SEviour, R. J. \& CODNER, R. C. (1973). Effect of light on carotenoid and riboflavin production by the fungus Cephalosporium diospyri. Journal of General Microbiology 77, 403-415.

THEIMER, R. R. \& RAU, W. (1970). Untersuchungen über die Lichtabhängige Carotenoidsynthese. V. Aufhebung der Lichtinduktion durch Reduktionsmittel und Ersatz des Lichts durch Wasserstoffperoxyd. Planta 92, I29-1 37.

VAlAdON, L. R. G. \& MUMmERY, R. S. (I97I $a$ ). Effect of light on nucleic acids, protein and carotenoids of Verticillum agaricinum. Microbios 4, 227-240.

Valadon, L. R. G. \& MummerY, R. S. (I97I $b$ ). Chromatography of carotenoids using papers filled with silica gel and with alumina. Phytochemistry Ir, 413-414. 